\title{
Editorial
}

\section{Mudança de Editor}

Após cerca de 10 anos à frente da RBEF, chegou a hora de passar o bastão para Silvio Dahmen e sua equipe, escolhidos pelo Conselho Deliberativo da SBF para dirigir a revista, pelo menos, no próximo triênio. Desejo-lhes muito sucesso.

A trajetória da RBEF durante o primeiro decênio do século XXI é bem conhecida e não tenho intenção neste momento de apresentar relatório das ações realizadas e metas alcançadas.

Desejo agradecer a todos os autores e colegas que acreditaram na RBEF submetendo artigos, apoiando a linha editorial, enviando sugestões, e, portanto, contribuindo para torná-la mais prestigiosa.

Ao final, meus sinceros agradecimentos aos colegas revisores, cuja lista de nomes segue abaixo, que me ajudaram efetivamente a manter um padrão de qualidade da RBEF.

Nelson Studart

Departamento de Física

Universidade Federal de São Carlos

Lista de revisores

Ademir Eugênio de Santana Ademir Eugênio de Santana Adenilson J. Chiquito Adilson J.A. de Oliveira Aguida M. Barreiro Alaor Chaves

Alberto Gaspar

Alberto Saa

Alberto Villani

Alex Bernardini

Alex G. Dias

Alexandre H. Andrei

Alexandre Tort

Alexys Bruno-Alfonso

Alice Pierson

Américo Tristão Bernardes

Amir O. Caldeira

Ana Maria Marques da Silva

André Ferrer P. Martins

André K.T. Assis

André Milone

Andreia Guerra

Anna Maria Pessoa de Carvalho

Antonio Augusto Passos Videira

Antônio Carlos Fontes dos Santos

Antônio Carlos Hernandes

Antonio Carlos Pedroza

Antonio Delfino Júnior
Antônio F.R. de Toledo Piza

Antônio Flavio Licarião Nogueira

Antonio Lima Santos

Antonio Mario Magalhães

Antonio Sérgio Pires

Antonio Soares de Castro

Arden Zylbersztajn

Arlene Cristina Aguilar

Augusto Beléndez Vázquez

Augusto Damineli Neto

Áurea Vasconcelos

Basílio Baseia

Beatriz L.S. Barbuy

Bernard Lesche

Bernardo Ruegger Almeida Neves

Bruto Max Pimentel Escobar

Caio Lewenkopf

Carlos Alberto dos Santos

Carlos Alberto Olivieri

Carlos Alberto Pelá

Carlos Alexandre Wuensche

Carlos Chesman de Araújo Feitosa

Carlos D'Alkaine

Carlos Eduardo Aguiar

Carlos Eduardo Laburú

Carlos Ernesto Garrido Salmon

Carlos Farina

Carlos Fiolhais
Carlos Lenz Cesar

Carlos O. Escobar

Carlos S.O. Yokoi

Carola Dobrigkeit Chinellato

Cássio Costa Laranjeiras

Cecil Chow Robilotta

Celso Grebogi

Celso Luiz Lima

Celso Villas Bôas

Cesar Constantino

Cesar Rogerio de Oliveira

Christovam Mendonça Filho

Cibelle Celestino Silva

Cláudia Vilega Rodrigues

Cláudio Antônio Cardoso

Cláudio Elias da Silva

Cláudio Lenz Cesar

Cleber Renato Mendonça

Concesa Caballero

Creso Franco

Cristiane Tavolaro

Cristiano Mattos

Daniel Gil-Pérez.

Daniel Augusto Turolla Vanzela

Daniel Gil Pérez

Daniel Pereira

David Mendez Soares

Deise M. Vianna

Copyright by the Sociedade Brasileira de Física. Printed in Brazil. 
Delano Gobbi

Demétrio Delizoicov

Diógenes Galetti

Dirceu da Silva

Divanizia do Nascimento Souza

Dmitri Maximovitch Guitman

Douglas Soares Galvão

Ducinei Garcia

Eder Rezende Moraes

Eduardo Afonso Terrazzan

Eduardo C. Marino

Eduardo de Campos Valadares

Eduardo Ernesto Castellano

Eduardo Gueron

Elcio Abdalla

Eliane A. Veit

Elio Carlos Ricardo

Elmo Salomão Alves

Emerson J.V. de Passos

Emico Okuno

Enos Picazzio

Erika R.M. Andreeta

Erika Zimmermann

Erivaldo Montarroyos

Ernesto P. Borges

Euclydes Marega Jr.

Eugenio Maria de França Ramosa

Evaldo Curado

Evaldo Ribeiro

Fátima M. Mitsue Yasuoka

Felipe Tovar Falciano

Fernanda Ostermann

Fernando de Sousa Barros

Fernando Lang da Silveira

Fernando M. Araújo-Moreira

Fernando Moraes

Fernando Pelegrini

Fernando T.C. Brandt

Filadelfo Cardoso Santos

Flávia Rezende

Flávio Caldas da Cruz

Francisco Artur Brown Chaves

Francisco Carlos Nard

Francisco Caruso

Francisco Castilho Alcaraz

Francisco Catelli

Francisco Coutinho

Francisco das Chagas Marques

George Emmanuel Matsas

George G. Kleiman

Gerson Francisco

Gilberto de Martino Jannuzzi

Gilberto Medeiros Kremer

Gilmar Eugenio Marques

Giovani Lopes Vasconcelos

Giuliano Augusto Pavan Ribeiro

Guilherme Leal Ferreira

Gustavo Rigolin

Gustavo Rojas

Hans-Peter Grieneisen

Helena Caldas

Hélio Vasconcelos Fagundes
Heloisa Maria Bertol Domingues

Henrique Boschi Filho

Henrique Fleming

Henrique Lins e Barros

Henrique von Dreifus

Humberto Carmona

Humberto M. França

Ildeu de Castro Moreira

Ileana Greca

Inez Staciarini Batista

Ioav Waga

Iria Guerrini

Isabel G. Rodrigues Martins

Itzhak Roditi

Ivano Damião Soares

Ives S. Araújo

Jaime Fernando Villas da Rocha

Jaime Frejlich

Jayme Vaz Junior

Jayme Vicente de Luca Jr.

Jenaro Guisasola

Jenner Barreto Bastos Filho

Jesuina Lopes de Almeida Pacca

João Batista Garcia Canalle

João Carlos Costa dos Anjos

João José Caluzi

João Luiz Kohl Moreira

João Sampaio

João Zanetic

Jorge Castiñeiras

Jorge Hönel

José Abdalla-Helayël Neto

José Albino Aguiar

José André Perez Angotti

José Antonio Brum

José Antonio Salvador

José Carlos Egues de Menezes

José Carlos Sartorelli

José David M. Vianna

José Eduardo M. Hornos

José Evangelista Moreira

José Fernando Fontanari

José Fernando Moura Rocha

José Francisco Sampaio

José Henrique Vuolo

José J. Lunazzi

José M. David Vianna

José Maria Filardo Bassalo

José Maria Villas Bôas

José Marques Póvoa

José Pedro Donoso Gonzalez

José Pedro Rino

José Ricardo G. Mendonça

José Roberto dos Santos Politi

José Roberto Drugowich de Felício

José Roberto Ruggiero

José Soares de Andrade Jr.

José Wellington R. Tabosa

Josif Frenkel

Juan Carlos Ceballos

Juan Linares López

Julia Salinas
Jürgen Fritz Stilck

Kaline Coutinho

Karina Lupetti

Kazunori Watari

Kepler de Souza Oliveira Filho

Ladir Candido

Laércio Ferracioli

Laerte Sodré Jr.

Leandro R. Tessler

Leila Maria Beltramini

Liacir dos Santos Lucena

Lidério C. Ioriatti Junior

Liliana Diogo

Liliane Ventura

Lizete Orquiza de Carvalho

Lucia Helena Mascaro

Luciano da Fontoura Costa

Lucila Cescato

Lucio Hora Acioli

Luis A. O. Nunes

Luis Alberto Cury

Luis Carlos Bassalo Crispino

Luis Carlos Jafelice

Luis Eduardo M. Oliveira

Luis Gustavo Marcassa

Luis Navarro Veguillas

Luis Nunes de Oliveira

Luisa Scolfaro

Luiz Agostinho Ferreira

Luiz Fernando Ziebell

Luiz Gallisa Guimarães

Luiz O.Q. Peduzzi

Marcelo A. de F. Gomes

Marcelo Alves Barros

Marcelo Andrade de Filgueiras Gomes

Marcelo Camargo de Juli

Marcelo Knobel

Marcelo M.F. Saba

Marcelo Rebouças

Marcelo Sousa Assumpção

Marcia C. Barbosa

Marco Antonio Amato

Marco Antonio Moreira

Marco Aurélio Cattacin Kneipp

Marco Moriconi

Marcos Cesar de Oliveira

Marcos Cesar Danhoni Neves

Marcos Leodoro

Marcus A.M. de Aguiar

Marcus Bastos Lacerda Santos

Marcus Raimundo Vale

Maria Assunção F. da Silva Dias

Maria Carolina Nemes

Maria Cristina Batoni Abdalla

Maria de Fátima Oliveira Saraiva

Maria do Carmo de Sousa

Maria Helena Roxo Beltran

Maria José P. Monteiro de Almeida

Maria José S.P. Brasil

Maria Lucia Vital dos Santos Abib

Maria Terezinha Xavier Silva

Mariangela Tassinari de Figueiredo 
Marisa Almeida Cavalcante

Marta Feijó Barroso

Marta Pesa

Maurício D. Coutinho-Filho

Mauricio Ortiz Calvão

Mauricio Pamplona Pires

Maurício Pietrocola de Oliveira

Mauricio Tiomno Tolmasquim

Mauro Dória

Mauro S.D. Cattani

Mercè Izquierdo

Mickel Abreu de Ponte

Miguel Novak

Mikiya Muramatsu

Miled Moussa

Mônica A. Cotta

Mucio Amado Continentino

Murilo P. de Almeida

Nathan Jacob Berkovits

Nelson Fiedler-Ferrara

Nelson Vani Leister

Newton Carneiro Affonso da Costa

Ney Lemke

Nilson Marcos Dias Garcia

Nivaldo A. Lemos

Nivaldo L. Speziali

Norberto Cardoso Ferreira

Odete Pacubi Baierl Teixeira

Odim Mendes Jr.

Olival Freire Jr.

Oscar Bolina

Oscar Éboli

Osvaldo Baffa Filho

Osvaldo Pessoa Jr.

Oswaldo Massambani

Oswaldo Novais de Oliveira Jr.

Oto Néri Borges

Pablo Rubén Mariconda

Patricia Nicolucci

Patricio Anibal Letelier

Paulo Américo Maia Neto

Paulo Batista Ramos

Paulo Cesar Coelho Abrantes

Paulo Cesar de Camargo
Paulo F. Farinas

Paulo Henrique Dionisio

Paulo Machado Mors

Paulo Murilo C. Oliveira

Paulo Pureur Neto

Paulo Ricardo da Silva Rosa

Paulo Roberto Silveira Gomes

Paulo Sérgio Pizani

Pedro Luis Malagutti

Pedro Morettin

Pedro Ruiz Castell

Penha Maria Cardozo Dias

Peter Schulz

Piotr Trzesniak

Ramayana Gazzinelli

Raul José Donangelo

Regina Kawamura

Reinaldo Welti

Rejane Maria Ribeiro Teixeira

Renê Robert

Reva Garg

Ricardo Barthem

Ricardo Ferreira

Ricardo L. Viana

Rita Maria C. de Almeida

Roberto Andrade

Roberto de Andrade Martins

Roberto Luis Moreira

Roberto Nardi

Roberto Nicolau Onody

Roberto Paterlini

Roberto Ribeiro da Silva

Roberto Zilles

Rogério C.T. da Costa

Rogério Rosenfeld

Roland Koberle

Romero Tavares

Ronald C. Shellard

Rubens de Andrade Jr.

Ruth de Sousa Schneider

Ruynet Matos Filho

Said R. Rabbani

Said Salem-Sugui Jr.

Salomon Mizrahi
Samuel Simon Rodrigues

Sandra Sampaio Vianna

Sandro Silva Costa

Sergio B. Volchan

Sérgio Carlos Zílio

Sérgio L.A. de Queiroz

Sérgio Mergulhão

Shirley Gobara

Silvestre Ragusa

Silvio L.S. Cunha

Silvio Renato Dahmen

Silvio Roberto Salinas

Silvio Seno Chibeni

Sonia Krapas

Susana de Sousa Barros

Suzana Maria Moss de Oliveira

Sylvio Canuto

Tânia Tomé

Tarciso Borges

Thomaz Ghilardi Netto

Tomaz Catunda

Trieste Freire Ricci

Ulisses Azevedo Leitão

Vagner Bernal Barbeta

Vagner Eustáquio de Carvalho

Valério Kurak

Valter Luis Líbero

Vanderlei Bagnato

Vicente Pleitez

Victor de Oliveira Rivelles

Victor López Richard

Vilson Toni Zanchin

Vito Roberto Vanin

Wagner Assis Cangussu Passos

Wagner Figueiredo

Walter Filgueira de Azevedo Jr.

Walter J. Maciel

Walter Wreszinski

Wilson Aires Ortiz

Xavier Roque

Yassuko Hosoume

Yuriko Baldin 